\title{
A Review: Induced Pluripotent Stem Cells (IPSC) and Their Reprogramming Techniques
}

\author{
Anusha Sudulaguntla* \\ Department of Pharmacy Practice, Siddhartha Institute of Pharmaceutical Sciences, Jonnalagadda, India
}

Submission: February 08, 2018; Published: March 14, 2018

*Corresponding author: Anusha Sudulaguntla, Assistant Professor, Department of Pharmacy Practice, Siddhartha Institute of Pharmaceutical Sciences, Jonnalagadda, Narasaraopeta, Guntur-522601, Andhra Pradesh, India, Tel: 0091-9535509073; Email: anusha9073@gmail.com

Abstract

Objective: Stem cells have the property to regenerate and replicate in to desired cells. They have the property to regulate and function in order to help the patient's condition and modify the organ function. They do show their therapeutic efficacy by regenerative method of treatment, which makes them special from all other types of medications which happen to have different mechanism of actions.

Back Ground: It is the peculiar nature of stem cells which made the humans questions their ability to create and repair the damaged organs. Ability to create a tissue both in vivo and in vitro has been a great achievement in the human history of personalized medicine. It has given a greater opportunity in the field of science and regenerative medicine.

Keywords: Induced pluripotent stem cells; Regenerative medicine; Oct4; Sox2; Klf4; c-Myc

Abbreviations: ICM: Inner Cell Mass; IPSC: Induced Pluripotent Stem Cells; PB: Piggyback

\section{Introduction}

Human embryonic cells from normal human embryos have the out most outcomes in the field of regenerative medicine. These cells have ultimate potential nature to replicate in to any type of cells a patient requires. Though it has many controversies revolving around the possible collection procedures of the same, it has been an interesting journey finding out other type of stem cells used in present day's medicine. Embryonic cells have been the 1 st and foremost cells to be used in the field of regenerative medicine. Therapeutic outcomes of this particular type of cells have been always in demand to be studied by the young generation. Understanding the capability of our own body to reproduce and repair is an amazing property we humans don't understand completely yet. Helping our own tissues to generate our degenerated organs is what made personalized medicine to its present stage of today. We humans have the tendency to question and find the answers, and later come the part of acceptance. Even though it was ridicules to the society when they first learned about regenerative medicine, the acceptance in the society has improved a long way as it stands today. People consider stem cell therapy as an initial step for personalized medicine; they also see it like a safety deposit to the upcoming future. It has been an incredible journey of stem cells from embryonic stem cells to induced pluripotent stem cells [1].

Pluripotent cells have the at most capacity to regenerate and repair. Example of pluripotent stem cells are embryonic stem cells, they have the ability to self-renew, as well as to differentiate in to cells of all the three germ layers, which is the property that makes it significant for regenerative property in IPSC. The major ethical issue regarding the use of human embryonic cells from inner cell mass (ICM) of the 4-7 day old embryo which destroys the progressive and developing embryo. If the study and research of this particular type of cells is success in the field of regenerative medicine, then it would lead to a lot of improvement in both the cellular medicine and also can be used for drug testing.

Adult stem cells are organ specific and have high threshold towards one particular organ which makes the cells limit to that one function associated with that one particular organ. All these cells are taken from genetic matter and are used to regenerate cells and use them in therapies [2].

Induced pluripotent stem cells, the humans way of saying, regeneration can be possible with any type of cells. It is the most possible way for improving the acceptance of stem cells in the society. IPSC are being researched in full potential way, and been studied a lot to understand their possible outcomes along with clinical applications.

Reprogramming and regenerative techniques shows the possibility of decreasing the use of immunosuppressive therapy, and these cells were first created by Takahashi and Yamaka in 
the year 2006, they converted adult mouse fibroblast to IPSC through ectopic expression of a selected group of transcription factors (OCT4, SOX2, LIF4, c-Myc). Further research has proven that one factor among the four; Oct 4 can actually be used to create the pluripotent stem cells in both rats and humans.

Although Yamaka's factors are sufficient to reprogram the cells to pluripotent stem cells, there are studies which are carried out to add other additional factors to this property of genetic modification. NANOG, LIN28, SV40 large T antigen are further said to enhance the pace of the cellular reprogramming methods. They express few cell markers such as TRA-1-60/TRA1-81, SSEA4, which diminish after the differentiation [2]. Other factors like addition of supplementation and other additional factors like valproic acid is said to improve the efficacy in generating the desired cells from IPSC [3]. Other techniques like adding small molecules to the reprogramming cocktails like butyrate, vitamin $C$ and Utfl, that improve cell senescence can be used to enhance the desired effect or out come in experimental basis [4].

\section{Reprogramming Techniques}

\section{Non-Viral}

Non-Viral methods of reprogramming gene delivery has been successful with effective out comes in stem cells therapeutic out come. One of the techniques is nucleofection and non-viral polycistronic construction containing reprogramming gene sequences and subsequent excision of factors using Cre/loxP system. Repeated rounds of transferring of these substances improve the yield the IPSC without any evidence of genomic integration. Experiments on non-integrating episomal vectors have also succeeded in IPSC generation makes use of the piggyback (PB) transposon which has the ability to be excised from its integration site without changing the original DNA sequence [5].

\section{Viral}

There are various methods tried for the past few years to induce IPSC in somatic cells which include somatic cell transfer, cell fusion, reprogramming through cell extracts and direct reprogramming. Initially retroviruses were developed in order to induce these genes in to the nucleus. The most recent focus has however shifted to direct reprogramming, which include lent virus, adenovirus, plasmid transfection and the PB transposition system [6].

\section{Relationship between Ipsc and Donor Cells}

The relation between the donor cells IPSC plays a crucial role while transferring and using the cells for regenerative methods for an individual for therapeutic outcomes. HIPSC hold memory of their particular donor cells and identity of the donor [7]. These gene expressions can lead to evidence of patient's history and can be used to study genetics and gene related studied which might also help in using these cells for drug tolerance studies and so on, anything related to that particular individual. The outcomes are promising and they do have evidence for positive study feedbacks, which ultimately states that the relationship between the cells and the donor is significant, and is very helpful in studying patient's details to the basic level, leading to preventive studies and inhibitory studies, leading to decrease in the incidence rate of the disease [8].

\section{IPSC in Drug Discovery Programme}

Drug discovery process is time taking is done step by step, consent from patient to approval of protocol, then formulating the drug molecule, clinical trials and then releasing it in to the market. There is a protocol for everything, IPSC can be used for drug discovery process, like I mentioned earlier, and the study of cell to its basic level can be done using IPSC.

I. $1^{\text {st }}$, recruitment of a patient cohort, along with appropriate healthy controls.

II. $2^{\text {nd }}$, derivation of high-quality, thoroughly characterized IPSCs by a scalable process, and storage of these iPSCs in a well-annotated biobank that allows the tracking and retrieval of samples and accompanying medical information from a relational database.

III. $3^{\text {rd }}$, differentiation of patient-derived iPSCs into the key cell types that are affected in disease.

IV. $4^{\text {th }}$, discovery of a disease phenotype.

V. $5^{\text {th }}$, configuration of an assay (based on the disease phenotype) that is robust, scalable and amenable to automation in a medium- to high-throughput manner for screening. The ability to grow billions of high-quality cells in a reproducible manner is essential.

Difference between Embryonic Stem Cells and Induced Pluripotent Stem Cells

There are multiple differences between embryonic stem cells and induced pluripotent stem cells. Few are listed as follows (Figure 1)

a) IPSC exhibit epigenetic differences from ES cells that are caused by aberrant methylation during early passages.

b) IPSC harbor residual DNA methylation signatures, namely epigenetic memory characteristics of their somatic tissue of origin, which favors their differentiation along lineages related to the donor cell, while restricting alternative cell fates.

c) The blood forming potential of IPSC derived from early bone marrow cells is higher than that of the IPSC derived from neural progenitor cells, whereas the potential is the same between nuclear transfer-ES cells and fertilized embryo-derived stem cells [9].

d) It is also seen that in case of blood and keratinocyte derivatives the condition is the same. 
e) Epigenetic abnormalities that arise during early reprogramming are inherited by IPSC. These include hundreds of abnormal gene silencing events, patterns of aberrant responses to epigenetic-modifying drugs resembling those of cancer cells, and the presence of cancer- specific gene promoter DNA methylation alterations.

f) In demonstration it is stated that, in contrast to ES cell derivatives, abnormal gene expression in some cells differentiated from IPSC can induce T-cell dependent immune responses in synergetic recipients.

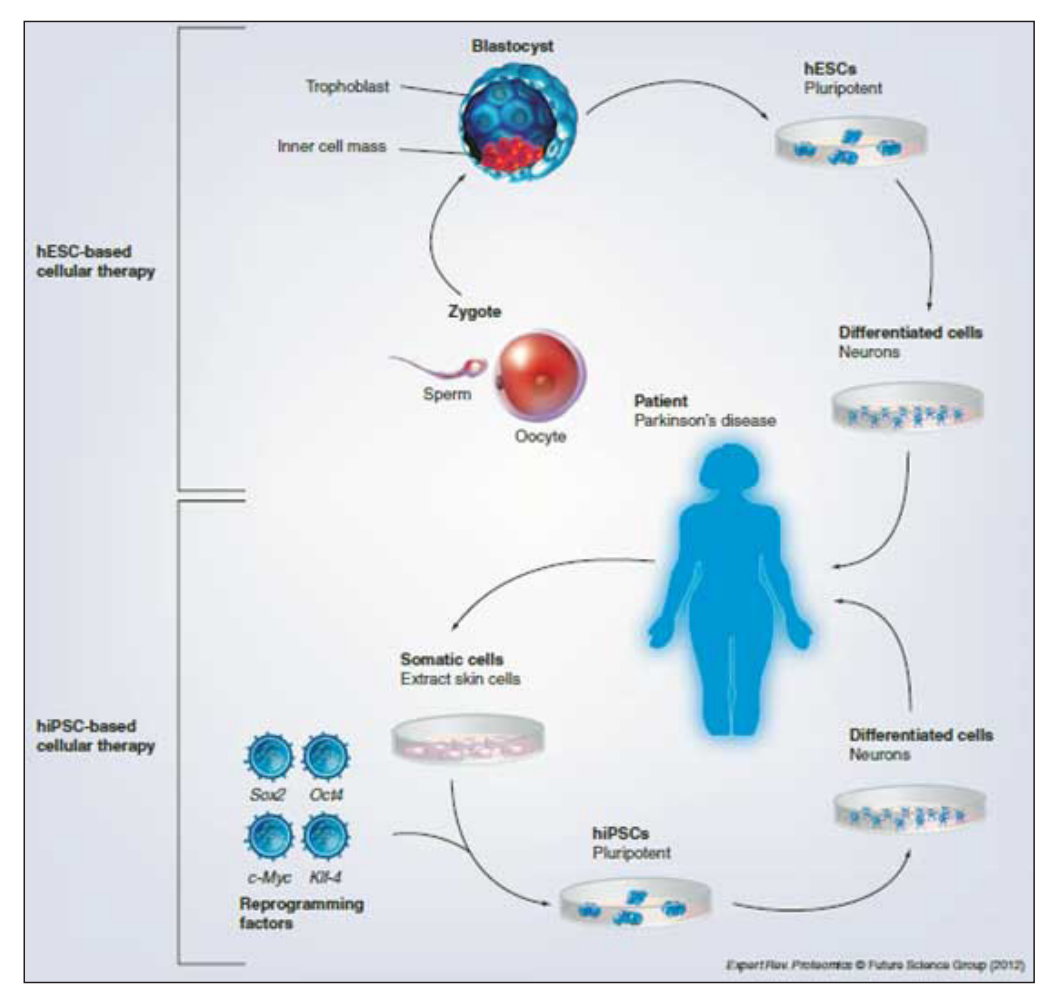

Figure 1: Difference between the two cellular therapies, on top is HUMAN EMBRYONIC STEM CELLS, below mentioned is Cultivation of IPSC

\section{Conclusion}

Induced pluripotent stem cells have the higher capacity to be practically used in personalized medicine, as they have abundant use and limited restrictions for their use. Stem cell therapy is the ultimate goal of personalized medicine and individual care for many diseases like degenerative Alzheimer's.

\section{References}

1. Pakzad M, Totonchi M, Taei A, Seifinejad A, Hassani SN, et al. (2009) Presence of a ROCK Inhibitor in Extracellular Matrix Supports More Undifferentiated Growth of Feeder-Free Human Embryonic and Induced Pluripotent Stem Cells upon Passaging. Stem Cell Rev and Rep 6: 96-107.

2. Ye Z, Cheng L (2010) Potential of human induced pluripotent stem cells derived from blood and other postnatal cell types. Regen Med 5(4): 521-530.

3. Jin ZB, Okamoto S, Mandai M, Takahashi M (2009) Induced pluripotent stem cells for retinal degenerative diseases: a new perspective on the challenges, Journal of Genetics 88(4): 417-424.
4. Lorenzo IM, Fleischer A, Bachiller D (2013) Generation of Mouse and Human Induced Pluripotent Stem Cells (iPSC) from Primary Somatic Cells. Stem Cell Rev and Rep 9: 435-450.

5. Rowntree RK, McNeish JD (2010) Induced pluripotent stem cells: opportunities as research and development tools in 21st century drug discovery. Regen Med 5(4): 557-568.

6. Walia B, Satija N, Tripathi RP, Gangenahalli GU (2012) Induced Pluripotent Stem Cells: Fundamentals and Applications of the Reprogramming Process and its Ramifications on Regenerative Medicine. Stem Cell Rev and Rep 8: 100-115.

7. Patel M, Yang S (2010) Advances in Reprogramming Somatic Cells to Induced Pluripotent Stem Cells. Stem Cell Rev and Rep 6: 367-380.

8. Cao L, Tan L, Jiang T, Zhu XC, Yu J (2011) Induced pluripotent stem cells, opportunities for disease modelling and drug discovery in neurodegenerative Diseases. Mol Neurobiol 10: 915-930.

9. Benevento M, Munoz J (2012) Role of mass spectrometry based proteomics in the study of cellular reprogramming and induced pluripotent stem cells. Expert Rev 9(4): 379-399. 
